PROCEEDINGS OF THE

AMERICAN MATHEMATICAL SOCIETY

Volume 136, Number 8, August 2008, Pages 2689-2698

S 0002-9939(08)09208-3

Article electronically published on April 8, 2008

\title{
SIGNED $q$-ANALOGS OF TORNHEIM'S DOUBLE SERIES
}

\author{
XIA ZHOU, TIANXIN CAI, AND DAVID M. BRADLEY
}

(Communicated by Ken Ono)

\begin{abstract}
We introduce signed $q$-analogs of Tornheim's double series and evaluate them in terms of double $q$-Euler sums. As a consequence, we provide explicit evaluations of signed and unsigned Tornheim double series and correct some mistakes in the literature.
\end{abstract}

\section{INTRODUCTION}

Let $k$ be a positive integer. Sums of the form (1)

$$
\zeta\left(s_{1}, s_{2}, \ldots, s_{k}\right):=\sum_{n_{1}>n_{2}>\cdots>n_{k}>0} \prod_{j=1}^{k} n_{j}^{-s_{j}}, \quad \sum_{j=1}^{m} \Re\left(s_{j}\right)>m, \quad m=1,2, \ldots, k,
$$

have attracted increasing attention in recent years; see e.g. 2, 3, 4, 5, 6, 8, 9, 10, 11, 13, 16, 24, 26, 27, 29, 30, 31. The survey articles $7,18,19,36,37,38$ provide an extensive list of references. In (11) the sum is over all positive integers $n_{1}, n_{2}, \ldots, n_{k}$ satisfying the indicated inequalities. Of course (1) reduces to the familiar Riemann zeta function when $k=1$. When the arguments are all positive integers, we refer to (11) as a multiple zeta value and note that in this case, $s_{1}>1$ is necessary and sufficient for convergence.

The problem of evaluating $\zeta\left(s_{1}, s_{2}\right)$ with integers $s_{1}>1$ and $s_{2}>0$ seems to have been first proposed in a letter from Goldbach to Euler 21] in 1742. (See also [20, 22] and [1, p. 253].) Calculating several examples led Euler to infer a closed form evaluation in terms of values of the Riemann zeta function in the case when $s_{1}+s_{2}$ is odd. In [4, Borwein, Bradley and Broadhurst considered the more general Euler sum

$$
\zeta\left(s_{1}, s_{2}, \ldots, s_{k} ; \sigma_{1}, \sigma_{2}, \ldots, \sigma_{k}\right):=\sum_{n_{1}>n_{2}>\cdots>n_{k}>0} \prod_{j=1}^{k} \sigma_{j}^{n_{j}} n_{j}^{-s_{j}}
$$

with each $\sigma_{j} \in\{-1,1\}$. Among the many other results for (2) listed therein is an explicit formula for the case $k=2$ that reduces to Euler's evaluation when $\sigma_{1}=$ $\sigma_{2}=1$. For the case of arbitrary $k$, several infinite classes of closed form evaluations

Received by the editors January 19, 2007, and in revised form April 25, 2007.

2000 Mathematics Subject Classification. Primary 11M41; Secondary 11M06, 05A30, 33E20, $30 \mathrm{~B} 50$.

Key words and phrases. Tornheim's double series, alternating Euler sums, multiple harmonic series, multiple zeta values, $q$-analog, $q$-series.

(C)2008 American Mathematical Society Reverts to public domain 28 years from publication 
for (11) and (2) are proved in [5, 6, 8, 9, 10. Subsequently, Bradley [12, 13, 14, 15] found $q$-analogs of (1) and (2) and thoroughly investigated their properties.

In 32 Tornheim introduced the double series

$$
T(r, s, t):=\sum_{u, v=1}^{\infty} \frac{1}{u^{r} v^{s}(u+v)^{t}},
$$

with nonnegative integers $r, s$ and $t$ satisfying $r+t>1, s+t>1$ and $r+s+$ $t>2$. Huard, Williams and Zhang [25] evaluated Tornheim's series in terms of sums of products of Riemann zeta values when $r+s+t$ is odd. Subbarao and Sitaramachandra Rao 28] considered the alternating variants

$$
R(r, s, t):=\sum_{u, v=1}^{\infty} \frac{(-1)^{v}}{u^{r} v^{s}(u+v)^{t}}, \quad S(r, s, t):=\sum_{u, v=1}^{\infty} \frac{(-1)^{u+v}}{u^{r} v^{s}(u+v)^{t}},
$$

and posed the problem to evaluate $R(r, r, r)$ and $S(r, r, r)$ for any positive integer $r$. Tsumura [33, Cor. 3] and [34, Theorem 3.6] tackled this problem and later [35] evaluated $S(r, s, t)$ for any positive integers $r, s, t$ such that $r+s+t$ is odd. Tsumura's method is elementary but complicated, and also has some mistakes. In this paper, we give a simple formula that expresses $R, S$ and $T$ in terms of double Euler sums. In light of the aforementioned formula of Borwein, Bradley and Broadhurst for the double Euler sums, our results yield a closed form evaluation for the series $R, S$ and $T$ whenever the arguments $r, s$ and $t$ are positive integers with $r+s+t$ odd. More generally, we consider $q$-analogs of $R, S, T$ and show how they may all be evaluated in terms of double $q$-Euler sums.

\section{2. $q$-ANALOGS}

Henceforth assume $q$ is real and $q>1$. The $q$-analog of a positive integer $n$ is

$$
[n]_{q}:=\sum_{j=0}^{n-1} q^{j}=\frac{q^{n}-1}{q-1} .
$$

Let $k$ be a positive integer, let $s_{1}, s_{2}, \ldots, s_{k}$ be real numbers, and let $\sigma_{1}, \sigma_{2}, \ldots, \sigma_{k} \in$ $\{-1,1\}$. Define the $q$-Euler sum

$$
\zeta_{q}\left[s_{1}, s_{2}, \ldots, s_{k} ; \sigma_{1}, \sigma_{2}, \ldots, \sigma_{k}\right]:=\sum_{n_{1}>n_{2}>\cdots>n_{k}>0} \prod_{j=1}^{k} \frac{\sigma_{j}^{n_{j}} q^{\left(s_{j}-1\right) n_{j}}}{\left[n_{j}\right]_{q}^{s_{j}}}
$$

and note that this coincides with the special case $\operatorname{Li}_{s_{1}, \ldots, s_{k}}\left[\sigma_{1} q^{s_{1}-1}, \ldots, \sigma_{k} q^{s_{k}-1}\right]$ of the multiple $q$-polylogarithm [12, eq. (6.2)]. If $\sigma_{j}=1$ for each $j=1,2, \ldots, k$, then we recover the multiple $q$-zeta value $\zeta\left[s_{1}, s_{2}, \ldots, s_{k}\right]$ of [12, 14, 15].

Let $\sigma, \tau \in\{-1,1\}$. Define $q$-analogs of the signed and unsigned Tornheim double series $R, S$ and $T$ by

$$
T[r, s, t ; \sigma, \tau]:=\sum_{u, v=1}^{\infty} \frac{\sigma^{u} \tau^{v} q^{(r+t-1) u+(s+t-1) v}}{[u]_{q}^{r}[v]_{q}^{s}[u+v]_{q}^{t}} .
$$

The sum

$$
\varphi[s ; \sigma]:=\sum_{n=1}^{\infty} \frac{(n-1) \sigma^{n} q^{(s-1) n}}{[n]_{q}^{s}}=\sum_{n=1}^{\infty} \frac{n \sigma^{n} q^{(s-1) n}}{[n]_{q}^{s}}-\zeta_{q}[s ; \sigma],
$$


the case $\sigma=1$ of which was defined in [15, will also be needed. As in 4, it is convenient to combine signs and exponents in (2) and (3) into a single list by writing $s_{j}$ if $\sigma_{j}=1$ and $\overline{s_{j}}$ if $\sigma_{j}=-1$. For consistency one may do this also for $T$ and $\varphi$; thus for example, $\varphi[s ; 1]=\varphi[s], \varphi[s ;-1]=\varphi[\bar{s}], R(r, s, t)=T(r, \bar{s}, t)$ and $S(r, s, t)=T(\bar{r}, \bar{s}, t)$. We also employ the notation

$$
\left(\begin{array}{c}
a+b+c \\
a, b
\end{array}\right):=\frac{(a+b+c) !}{a ! b ! c !}
$$

for the multinomial coefficient, in which $a, b$ and $c$ are all nonnegative integers. The following theorem shows how the $q$-analogs of $R, S$ and $T$ are related to the $q$-Euler sums.

Theorem 1. Let $r$ and $s$ be positive integers, and let $t$ be a real number. Then

$$
\begin{aligned}
T[r, s, t] & =\sum_{a=0}^{r-1} \sum_{b=0}^{r-1-a}\left(\begin{array}{c}
a+s-1 \\
a, b
\end{array}\right)(1-q)^{b} \zeta_{q}[s+t+a, r-a-b] \\
& +\sum_{a=0}^{s-1} \sum_{b=0}^{s-1-a}\left(\begin{array}{c}
a+r-1 \\
a, b
\end{array}\right)(1-q)^{b} \zeta_{q}[r+t+a, s-a-b] \\
& -\sum_{j=1}^{\min (r, s)}\left(\begin{array}{c}
r+s-j-1 \\
r-j, s-j
\end{array}\right)(1-q)^{j} \varphi[r+s+t-j], \\
T[\bar{r}, \bar{s}, t]= & \sum_{a=0}^{r-1} \sum_{b=0}^{r-1-a}\left(\begin{array}{c}
a+s-1 \\
a, b
\end{array}\right)(1-q)^{b} \zeta_{q}[\overline{s+t+a}, r-a-b] \\
& +\sum_{a=0}^{s-1} \sum_{b=0}^{s-1-a}\left(\begin{array}{c}
a+r-1 \\
a, b
\end{array}\right)(1-q)^{b} \zeta_{q}[\overline{r+t+a}, s-a-b] \\
& -\sum_{j=1}^{\min (r, s)}\left(\begin{array}{c}
r+s-j-1 \\
r-j, s-j
\end{array}\right)(1-q)^{j} \varphi_{[}[\overline{r+s+t-j}], \\
T[r, \bar{s}, t]= & \sum_{a=0}^{r-1} \sum_{b=0}^{r-1-a}\left(\begin{array}{c}
a+s-1 \\
a, b
\end{array}\right)(1-q)^{b} \zeta_{q}[\overline{s+t+a}, \overline{r-a-b}] \\
& +\sum_{a=0}^{s-1} \sum_{b=0}^{s-1-a}\left(\begin{array}{c}
a+r-1 \\
a, b
\end{array}\right)(1-q)^{b} \zeta_{q}[r+t+a, \overline{s-a-b}] \\
& -\sum_{j=1}^{\min (r, s)}\left(\begin{array}{c}
r+s-j-1 \\
r-j, s-j
\end{array}\right)(1-q)^{j}(1+q)^{j-r-s-t} \zeta_{q^{2}}[r+s+t-j] .
\end{aligned}
$$

Taking the limit as $q \rightarrow 1+$ in Theorem 1 and noting the restrictions on $r, s$ and $t$ now needed for convergence yield the following.

Corollary 1. Let $r$ and $s$ be positive integers and let $t$ be a real number. If $r+t>1$ and $s+t>1$, then

$$
T(r, s, t)=\sum_{a=0}^{r-1}\left(\begin{array}{c}
a+s-1 \\
s-1
\end{array}\right) \zeta(s+t+a, r-a)+\sum_{a=0}^{s-1}\left(\begin{array}{c}
a+r-1 \\
r-1
\end{array}\right) \zeta(r+t+a, s-a) ;
$$


if $r+t>0$ and $s+t>0$, then

$S(r, s, t)=\sum_{a=0}^{r-1}\left(\begin{array}{c}a+s-1 \\ s-1\end{array}\right) \zeta(\overline{s+t+a}, r-a)+\sum_{a=0}^{s-1}\left(\begin{array}{c}a+r-1 \\ r-1\end{array}\right) \zeta(\overline{r+t+a}, s-a) ;$

if $r+t>1$ and $s+t>0$, then

$R(r, s, t)=\sum_{a=0}^{r-1}\left(\begin{array}{c}a+s-1 \\ s-1\end{array}\right) \zeta(\overline{s+t+a}, \overline{r-a})+\sum_{a=0}^{s-1}\left(\begin{array}{c}a+r-1 \\ r-1\end{array}\right) \zeta(r+t+a, \overline{s-a})$.

Putting $t=0$ in Theorem 1 yields the following decomposition formulas, the first of which was given under slightly more restrictive hypotheses in [15, Theorem 2.1].

Corollary 2. If $r$ and $s$ are positive integers, then

$$
\begin{aligned}
& \zeta_{q}[r] \zeta_{q}[s]=\sum_{a=0}^{r-1} \sum_{b=0}^{r-1-a}\left(\begin{array}{c}
a+s-1 \\
s-1
\end{array}\right)\left(\begin{array}{c}
s-1 \\
b
\end{array}\right)(1-q)^{b} \zeta_{q}[s+a, r-a-b] \\
& +\sum_{a=0}^{s-1} \sum_{b=0}^{s-1-a}\left(\begin{array}{c}
a+r-1 \\
r-1
\end{array}\right)\left(\begin{array}{c}
r-1 \\
b
\end{array}\right)(1-q)^{b} \zeta_{q}[r+a, s-a-b] \\
& -\sum_{j=1}^{\min (r, s)}\left(\begin{array}{c}
r+s-j-1 \\
r-j, s-j
\end{array}\right)(1-q)^{j} \varphi[r+s-j] \text {; } \\
& \zeta_{q}[\bar{r}] \zeta_{q}[\bar{s}]=\sum_{a=0}^{r-1} \sum_{b=0}^{r-1-a}\left(\begin{array}{c}
a+s-1 \\
s-1
\end{array}\right)\left(\begin{array}{c}
s-1 \\
b
\end{array}\right)(1-q)^{b} \zeta_{q}[\overline{s+a}, r-a-b] \\
& +\sum_{a=0}^{s-1} \sum_{b=0}^{s-1-a}\left(\begin{array}{c}
a+r-1 \\
r-1
\end{array}\right)\left(\begin{array}{c}
r-1 \\
b
\end{array}\right)(1-q)^{b} \zeta_{q}[\overline{r+a}, s-a-b] \\
& -\sum_{j=1}^{\min (r, s)}\left(\begin{array}{c}
r+s-j-1 \\
r-j, s-j
\end{array}\right)(1-q)^{j} \varphi[\overline{r+s-j}] \text {; } \\
& \zeta_{q}[r] \zeta_{q}[\bar{s}]=\sum_{a=0}^{r-1} \sum_{b=0}^{r-1-a}\left(\begin{array}{c}
a+s-1 \\
s-1
\end{array}\right)\left(\begin{array}{c}
s-1 \\
b
\end{array}\right)(1-q)^{b} \zeta_{q}[\overline{s+a}, \overline{r-a-b}] \\
& +\sum_{a=0}^{s-1} \sum_{b=0}^{s-1-a}\left(\begin{array}{c}
a+r-1 \\
r-1
\end{array}\right)\left(\begin{array}{c}
r-1 \\
b
\end{array}\right)(1-q)^{b} \zeta_{q}[r+a, \overline{s-a-b}] \\
& -\sum_{j=1}^{\min (r, s)}\left(\begin{array}{c}
r+s-j-1 \\
r-j, s-j
\end{array}\right)(1-q)^{j}(1+q)^{j-r-s} \zeta_{q^{2}}[r+s-j] .
\end{aligned}
$$

Taking the limit as $q \rightarrow 1+$ in Corollary 2 and noting the additional restrictions needed on $r$ and $s$ to guarantee convergence in this case yield the following decomposition formulas, the first of which was known to Euler.

Corollary 3. If $r-1$ and $s-1$ are positive integers, then

$$
\zeta(r) \zeta(s)=\sum_{a=0}^{r-1}\left(\begin{array}{c}
a+s-1 \\
s-1
\end{array}\right) \zeta(s+a, r-a)+\sum_{a=0}^{s-1}\left(\begin{array}{c}
a+r-1 \\
r-1
\end{array}\right) \zeta(r+a, s-a) ;
$$


if $r$ and $s$ are positive integers, then

$$
\zeta(\bar{r}) \zeta(\bar{s})=\sum_{a=0}^{r-1}\left(\begin{array}{c}
a+s-1 \\
s-1
\end{array}\right) \zeta(\overline{s+a}, r-a)+\sum_{a=0}^{s-1}\left(\begin{array}{c}
a+r-1 \\
r-1
\end{array}\right) \zeta(\overline{r+a}, s-a)
$$

if $r-1$ and $s$ are positive integers, then

$$
\zeta(r) \zeta(\bar{s})=\sum_{a=0}^{r-1}\left(\begin{array}{c}
a+s-1 \\
s-1
\end{array}\right) \zeta(\overline{s+a}, \overline{r-a})+\sum_{a=0}^{s-1}\left(\begin{array}{c}
a+r-1 \\
r-1
\end{array}\right) \zeta(r+a, \overline{s-a}) .
$$

\section{Proof of Theorem 1}

The key ingredient is the following partial fraction decomposition.

Lemma 1. If $r, s, u$, and $v$ are all positive integers, then

$$
\begin{aligned}
\frac{1}{[u]_{q}^{r}[v]_{q}^{s}} & =\sum_{a=0}^{r-1} \sum_{b=0}^{r-1-a}\left(\begin{array}{c}
a+s-1 \\
a, b
\end{array}\right) \frac{(1-q)^{b} q^{(s-1-b) u+a v}}{[u]_{q}^{r-a-b}[u+v]_{q}^{s+a}} \\
& +\sum_{a=0}^{s-1} \sum_{b=0}^{s-1-a}\left(\begin{array}{c}
a+r-1 \\
a, b
\end{array}\right) \frac{(1-q)^{b} q^{a u+(r-1-b) v}}{[v]_{q}^{s-a-b}[u+v]_{q}^{r+a}} \\
& -\sum_{j=1}^{\min (r, s)}\left(\begin{array}{c}
r+s-j-1 \\
r-j, s-j
\end{array}\right) \frac{(1-q)^{j} q^{(s-j) u+(r-j) v}}{[u+v]_{q}^{r+s-j}} .
\end{aligned}
$$

Proof. Let $x$ and $y$ be nonzero real numbers such that $x+y+(q-1) x y \neq 0$. As in [15], observe that if we apply the partial differential operator

$$
\frac{1}{(r-1) !}\left(-\frac{\partial}{\partial x}\right)^{r-1} \frac{1}{(s-1) !}\left(-\frac{\partial}{\partial y}\right)^{s-1}
$$

to both sides of the identity

$$
\frac{1}{x y}=\frac{1}{x+y+(q-1) x y}\left(\frac{1}{x}+\frac{1}{y}+q-1\right),
$$

then we obtain the identity [15, Lemma 3.1]

$$
\begin{aligned}
\frac{1}{x^{r} y^{s}} & =\sum_{a=0}^{r-1} \sum_{b=0}^{r-1-a}\left(\begin{array}{c}
a+s-1 \\
a, b
\end{array}\right) \frac{(1-q)^{b}(1+(q-1) y)^{a}(1+(q-1) x)^{s-1-b}}{x^{r-a-b}(x+y+(q-1) x y)^{s+a}} \\
& +\sum_{a=0}^{s-1} \sum_{b=0}^{s-1-a}\left(\begin{array}{c}
a+r-1 \\
a, b
\end{array}\right) \frac{(1-q)^{b}(1+(q-1) x)^{a}(1+(q-1) y)^{r-1-b}}{y^{s-a-b}(x+y+(q-1) x y)^{r+a}} \\
& -\sum_{j=1}^{\min (r, s)}\left(\begin{array}{c}
r+s-j-1 \\
r-j, s-j
\end{array}\right) \frac{(1-q)^{j}(1+(q-1) y)^{r-j}(1+(q-1) x)^{s-j}}{(x+y+(q-1) x y)^{r+s-j}} .
\end{aligned}
$$

Now let $x=[u]_{q}, y=[v]_{q}$ and note that then $1+(q-1) x=q^{u}, 1+(q-1) y=q^{v}$ and $x+y+(q-1) x y=[u+v]_{q}$.

To prove Theorem 1, multiply both sides of Lemma 1 by

$$
\frac{\sigma^{u} \tau^{v} q^{(r+t-1) u+(s+t-1) v}}{[u+v]_{q}^{t}}
$$


and sum over all ordered pairs of positive integers $(u, v)$ to obtain

$$
\begin{aligned}
T[r, s, t ; \sigma, \tau] & =\sum_{a=0}^{r-1} \sum_{b=0}^{r-1-a}\left(\begin{array}{c}
a+s-1 \\
a, b
\end{array}\right)(1-q)^{b} \sum_{u, v=1}^{\infty} \frac{\sigma^{u} \tau^{v} q^{(r-a-b-1) u} q^{(s+t+a-1)(u+v)}}{[u]_{q}^{r-a-b}[u+v]_{q}^{s+t+a}} \\
& +\sum_{a=0}^{s-1} \sum_{b=0}^{s-1-a}\left(\begin{array}{c}
a+r-1 \\
a, b
\end{array}\right)(1-q)^{b} \sum_{u, v=1}^{\infty} \frac{\sigma^{u} \tau^{v} q^{(s-a-b-1) v} q^{(r+t+a-1)(u+v)}}{[v]_{q}^{s-a-b}[u+v]_{q}^{r+t+a}} \\
& -\sum_{j=1}^{\min (r, s)}\left(\begin{array}{c}
r+s-j-1 \\
r-j, s-j
\end{array}\right)(1-q)^{j} \sum_{u, v=1}^{\infty} \frac{\sigma^{u} \tau^{v} q^{(r+s+t-j-1)(u+v)}}{[u+v]_{q}^{r+s+t-j}}
\end{aligned}
$$

It follows that

$$
\begin{aligned}
T[r, s, t ; \sigma, \sigma] & =\sum_{a=0}^{r-1} \sum_{b=0}^{r-1-a}\left(\begin{array}{c}
a+s-1 \\
a, b
\end{array}\right)(1-q)^{b} \sum_{m>n>0} \frac{\sigma^{m} q^{(s+t+a-1) m} q^{(r-a-b-1) n}}{[m]_{q}^{s+t+a}[n]_{q}^{r-a-b}} \\
& +\sum_{a=0}^{s-1} \sum_{b=0}^{s-1-a}\left(\begin{array}{c}
a+r-1 \\
a, b
\end{array}\right)(1-q)^{b} \sum_{m>n>0} \frac{\sigma^{m} q^{(r+t+a-1) m} q^{(s-a-b-1) n}}{[m]^{r+t+a}[n]_{q}^{s-a-b}} \\
& -\sum_{j=1}^{\min (r, s)}\left(\begin{array}{c}
r+s-j-1 \\
r-j, s-j
\end{array}\right)(1-q)^{j} \sum_{m>n>0} \frac{\sigma^{m} q^{(r+s+t-j-1) m}}{[m]_{q}^{r+s+t-j}} \\
& =\sum_{a=0}^{r-1} \sum_{b=0}^{r-1-a}\left(\begin{array}{c}
a+s-1 \\
a, b
\end{array}\right)(1-q)^{b} \zeta_{q}[s+t+a, r-a-b ; \sigma, 1] \\
& +\sum_{a=0}^{s-1} \sum_{b=0}^{s-1-a}\left(\begin{array}{c}
a+r-1 \\
a, b
\end{array}\right)(1-q)^{b} \zeta_{q}[r+t+a, s-a-b ; \sigma, 1] \\
& -\sum_{j=1}^{\min (r, s)}\left(\begin{array}{c}
r+s-j-1 \\
r-j, s-j
\end{array}\right)(1-q)^{j} \varphi[r+s+t-j ; \sigma],
\end{aligned}
$$

and also that

$$
\begin{aligned}
T[r, s, t ; 1,-1] & =\sum_{a=0}^{r-1} \sum_{b=0}^{r-1-a}\left(\begin{array}{c}
a+s-1 \\
a, b
\end{array}\right)(1-q)^{b} \\
\times \sum_{m>n>0} \frac{(-1)^{m} q^{(s+t+a-1) m}(-1)^{n} q^{(r-a-b-1) n}}{[m]_{q}^{s+t+a}[n]_{q}^{r-a-b}} & \\
& +\sum_{a=0}^{s-1} \sum_{b=0}^{s-1-a}\left(\begin{array}{c}
a+r-1 \\
a, b
\end{array}\right)(1-q)^{b} \sum_{m>n>0} \frac{q^{(r+t+a-1) m}(-1)^{n} q^{(s-a-b-1) n}}{[m]_{q}^{r+t+a}[n]_{q}^{s-a-b}} \\
& -\sum_{j=1}^{\min (r, s)}\left(\begin{array}{c}
r+s-j-1 \\
r-j, s-j
\end{array}\right)(1-q)^{j} \sum_{m>n>0} \frac{(-1)^{n} q^{(r+s+t-j-1) m}}{[m]_{q}^{r+s+t-j}} \\
& =\sum_{a=0}^{r-1} \sum_{b=0}^{r-1-a}\left(\begin{array}{c}
a+s-1 \\
a, b
\end{array}\right)(1-q)^{b} \zeta_{q}[s+t+a, r-a-b ; \sigma, 1]
\end{aligned}
$$




$$
\begin{aligned}
& +\sum_{a=0}^{s-1} \sum_{b=0}^{s-1-a}\left(\begin{array}{c}
a+r-1 \\
a, b
\end{array}\right)(1-q)^{b} \zeta_{q}[r+t+a, s-a-b ; \sigma, 1] \\
& +\sum_{j=1}^{\min (r, s)}\left(\begin{array}{c}
r+s-j-1 \\
r-j, s-j
\end{array}\right)(1-q)^{j} \sum_{m>0} \frac{q^{(r+s+t-j-1) 2 m}}{[2 m]_{q}^{r+s+t-j}} .
\end{aligned}
$$

Since

$$
[2 m]_{q}=\frac{q^{2 m}-1}{q-1}=\frac{q^{2}-1}{q-1} \cdot \frac{q^{2 m}-1}{q^{2}-1}=(q+1)[m]_{q^{2}},
$$

the proof of Theorem 1 is complete.

\section{EXAMPLES}

Again, let $\sigma, \tau \in\{-1,1\}$. It is known [4, 23] that if $s$ and $t$ are positive integers such that $s+t$ is odd and $s>(1+\sigma) / 2$, then $\zeta(s, t ; \sigma, \tau)$ lies in the polynomial $\operatorname{ring} \mathbf{Q}[\{\zeta(k ; \pm 1): k \in \mathbf{Z}, 2 \leq k \leq s+t\} \cup\{\zeta(1 ;-1)\}]$. Since

$$
\zeta(k ;-1)=\zeta(\bar{k})= \begin{cases}\left(2^{1-k}-1\right) \zeta(k) & \text { if } k>1, \\ -\log 2 & \text { if } k=1,\end{cases}
$$

it is clear that in fact, $\zeta(s, t ; \sigma, \tau) \in \mathbf{Q}[\{\zeta(k): k \in \mathbf{Z}, 2 \leq k \leq s+t\} \cup\{-\log 2\}]$. It follows that if $r, s, t$ satisfy the conditions of Corollary 1 and if in addition $r+s+t$ is an odd integer, then the signed and unsigned double Tornheim series $R(r, s, t)$, $S(r, s, t)$ and $T(r, s, t)$ also lie in this ring. It is possible to evaluate these series explicitly if we recall the following formula from [4, eq. (75)].

Proposition 1. Let $\sigma, \tau \in\{-1,1\}$, and let $s$ and $t$ be positive integers such that $s+t$ is odd, $s>(1+\sigma) / 2$, and $t>(1+\tau) / 2$. Then

$$
\begin{aligned}
& \zeta(s, t ; \sigma, \tau)=\frac{1}{2}\left(1+(-1)^{s}\right) \zeta(s ; \sigma) \zeta(t ; \tau)-\frac{1}{2} \zeta(s+t ; \sigma \tau) \\
& +(-1)^{t} \sum_{0 \leq k \leq t / 2}\left(\begin{array}{c}
s+t-2 k-1 \\
s-1
\end{array}\right) \zeta(2 k ; \sigma \tau) \zeta(s+t-2 k ; \sigma) \\
& +(-1)^{t} \sum_{0 \leq k \leq s / 2}\left(\begin{array}{c}
s+t-2 k-1 \\
t-1
\end{array}\right) \zeta(2 k ; \sigma \tau) \zeta(s+t-2 k ; \tau) .
\end{aligned}
$$

In Proposition 1, it is understood that $\zeta(0 ; \sigma \tau)=-1 / 2$ in accordance with the analytic continuation of $s \mapsto \zeta(s ; \sigma \tau)$. The restriction $t>(1+\tau) / 2$ can be removed if in (44) we interpret $\zeta(1 ; 1)=0$ wherever it occurs. That is, if $\sigma \in\{-1,1\}$ and $s$ is an even positive integer, then

(5) $\zeta(s, 1 ; \sigma, 1)=\frac{1}{2}(s-1) \zeta(s+1 ; \sigma)+\frac{1}{2} \zeta(s+1)-\sum_{k=1}^{(s / 2)-1} \zeta(2 k ; \sigma) \zeta(s+1-2 k)$.

The case $\sigma=1$ of (5) is subsumed by another formula [4, eq. (31)] of Euler, namely

$$
\zeta(s, 1)=\frac{s}{2} \zeta(s+1)-\frac{1}{2} \sum_{k=2}^{s-1} \zeta(k) \zeta(s+1-k),
$$

which is valid for all integers $s>1$, not just for even $s$.

In [35, Tsumura listed evaluation formulas for $S(r, s, t)$ when $r+s+t \leq 9$ is odd. From Corollary 1, Proposition 1 and equation (5), we can deduce explicit 
formulas for $R(r, s, t), S(r, s, t)$ and $T(r, s, t)$ when $r+s+t$ is odd. In particular, we have the following new results:

$$
\begin{aligned}
& R(1,1,1)=-\frac{5}{8} \zeta(3), \\
& R(1,1,3)=\frac{1}{16} \pi^{2} \zeta(3)-\frac{27}{32} \zeta(5), \\
& R(1,2,2)=\frac{5}{48} \pi^{2} \zeta(3)-\frac{3}{2} \zeta(5), \\
& R(1,3,1)=\frac{1}{12} \pi^{2} \zeta(3)-\frac{59}{32} \zeta(5), \\
& R(2,1,2)=-\frac{5}{16} \pi^{2} \zeta(3)+\frac{107}{32} \zeta(5), \\
& R(2,2,1)=-\frac{5}{24} \pi^{2} \zeta(3)+\frac{59}{32} \zeta(5), \\
& R(3,1,1)=\frac{1}{8} \pi^{2} \zeta(3)-\frac{59}{32} \zeta(5), \\
& S(5,5,5)=\frac{7}{73728} \pi^{4} \zeta(11)+\frac{35}{24576} \pi^{2} \zeta(13)+\frac{63}{8192} \zeta(15), \\
& S(7,7,7)=\frac{31}{35389440} \pi^{6} \zeta(15)+\frac{49}{1966080} \pi^{4} \zeta(17)+\frac{77}{262144} \pi^{2} \zeta(19)+\frac{429}{262144} \zeta(21)
\end{aligned}
$$

The values of $R(5,5,5), R(7,7,7)$ and $R(9,9,9)$ listed in 34 appear to be incorrect. They should be

$$
\begin{aligned}
& R(5,5,5)=\frac{16375}{147456} \pi^{4} \zeta(11)+\frac{573335}{49152} \pi^{2} \zeta(13)-\frac{2064195}{16384} \zeta(15), \\
& R(7,7,7)=\frac{1048543}{70778880} \pi^{6} \zeta(15)+\frac{7339969}{3932160} \pi^{4} \zeta(17)+\frac{80740121}{524288} \pi^{2} \zeta(19) \\
&-\frac{899676921}{524288} \zeta(21), \\
& R(9,9,9)=\frac{13421747}{7046430720} \pi^{8} \zeta(19)+\frac{738197141}{2113929216} \pi^{6} \zeta(21)+\frac{1919313253}{67108864} \pi^{4} \zeta(23) \\
&+ \frac{143948506845}{67108864} \pi^{2} \zeta(25)-\frac{1631416447375}{67108864} \zeta(27) . \\
& \text { REFERENCES }
\end{aligned}
$$

\section{REFERENCES}

[1] B. Berndt, Ramanujan's Notebooks. Part I, Springer-Verlag, New York, 1985. MR0781125 (86c:01062)

[2] D. Borwein, J. M. Borwein, and D. M. Bradley, Parametric Euler sum identities, J. Math. Anal. Appl., 316 (2006), no. 1, 328-338. MR2201764(2007b:11132)

[3] J. M. Borwein and D. M. Bradley, Thirty-two Goldbach variations, Internat. J. Number Theory, 2 (2006), no. 1, 65-103. MR2217795 (2007e:11109)

[4] J. M. Borwein, D. M. Bradley, and D. J. Broadhurst, Evaluations of $k$-fold Euler/Zagier sums: A compendium of results for arbitrary $k$, Electron. J. Combin., 4 (1997), no. 2, \#R5. Wilf Festschrift. MR1444152 (98b:11091)

[5] J. M. Borwein, D. M. Bradley, D. J. Broadhurst, and P. Lisoněk, Combinatorial aspects of multiple zeta values, Electron. J. Combin., 5 (1998), no. 1, \#R38. MR1637378 (99g:11100)

[6] Special values of multiple polylogarithms, Trans. Amer. Math. Soc., 353 (2001), no. 3, 907-941. MR 1709772 (2003i:33003)

[7] D. Bowman and D. M. Bradley, Multiple polylogarithms: A brief survey, Proceedings of a Conference on $q$-Series with Applications to Combinatorics, Number Theory and Physics (B. C. Berndt and K. Ono, eds., Urbana, IL, 2000), Contemporary Math., 291, Amer. Math. Soc., Providence, RI, 2001, pp. 71-92. MR1874522 (2003c:33021) 
[8] The algebra and combinatorics of shuffles and multiple zeta values, J. Combin. Theory Ser. A, 97 (2002), no. 1, 43-61. MR.1879045(2003j:05010)

[9] Resolution of some open problems concerning multiple zeta evaluations of arbitrary depth, Compositio Math., 139 (2003), no. 1, 85-100. MR2024966 (2005f:11196)

[10] D. Bowman, D. M. Bradley, and J. Ryoo, Some multi-set inclusions associated with shuffle convolutions and multiple zeta values, European J. Combin., 24 (2003), no. 1, 121-127. MR:1957970 (2004c:11115)

[11] D. M. Bradley, Partition identities for the multiple zeta function, Zeta Functions, Topology, and Quantum Physics, Developments in Mathematics, 14, T. Aoki, S. Kanemitsu, M. Nakahara, Y. Ohno (eds.), Springer, New York, 2005, pp. 19-29. MR2179270 (2006f:11105)

[12] _. Multiple q-zeta values, J. Algebra, 283 (2005), no. 2, 752-798. MR2111222 (2006f:11106)

[13] , Duality for finite multiple harmonic $q$-series, Discrete Math., 300 (2005), no. 1-3, 44-56. MR2170113(2006m:05019)

[14] _ On the sum formula for multiple $q$-zeta values, Rocky Mountain J. Math., to appear. http://arxiv.org/abs/math.QA/0411274

[15] _ A $q$-analog of Euler's decomposition formula for the double zeta function, Internat. J. Math. Math. Sci., 2005, no. 21, 3453-3458. MR2206867 (2006k:11174)

[16] D. J. Broadhurst and D. Kreimer, Association of multiple zeta values with positive knots via Feynman diagrams up to 9 loops, Phys. Lett. B, 393 (1997), no. 3-4, 403-412. MR1435933 (98g:11101)

[17] F. C. Brown, Périodes des espaces des modules $\overline{\mathfrak{M}}_{0, n}$ et valeurs zêtas multiples [Multiple zeta values and periods of the moduli spaces $\left.\overline{\mathfrak{M}}_{0, n}\right]$, C. R. Math. Acad. Sci. Paris, 342 (2006), no. 12, 949-954. MR2235616

[18] P. Cartier, Fonctions polylogarithmes, nombres polyzêtas et groupes pro-unipotents, Astérisque, 282 (2002), viii, 137-173, Séminaire Bourbaki, 53iéme année, 2000-2001, Exp. No. 885. MR1975178 (2004i:19005)

[19] - Values of the $\zeta$-function, Surveys in Modern Mathematics, 260-273, London Math. Soc. Lecture Note Ser., 321, Cambridge Univ. Press, Cambridge, 2005. MR2166932 (2006g:11182)

[20] L. Euler, Meditationes Circa Singulare Serierum Genus, Novi Comm. Acad. Sci. Petropol., 20 (1775), 140-186. Reprinted in "Opera Omnia," ser. I, 15, B. G. Teubner, Berlin (1927), $217-267$.

[21] , Briefwechsel, vol. 1, Birkhäuser, Basel, 1975. MR0497632

[22] L. Euler and C. Goldbach, Briefwechsel 1729-1764, Akademie-Verlag, Berlin, 1965.

[23] P. Flajolet and B. Salvy, Euler sums and contour integral representations, Experiment. Math., 7 (1998), no. 1, 15-35. MR.1618286 (99c:11110)

[24] M. E. Hoffman, Periods of mirrors and multiple zeta values, Proc. Amer. Math. Soc., 130 (2002), no. 4, 971-974. MR 1873769 (2002k:14068)

[25] J. G. Huard, K. S. Williams, and N.-Y. Zhang, On Tornheim's double series, Acta Arith., 75 (1996), no. 2, 105-117. MR1379394 (97f:11073)

[26] K. Ihara and T. Takamuki, The quantum $\mathfrak{g}_{2}$ invariant and relations of multiple zeta values, J. Knot Theory Ramifications, 10 (2001), no. 7, 983-997. MR.1867104(2002m:57016)

[27] T. Q. T. Le and J. Murakami, Kontsevich's integral for the Homfly polynomial and relations between values of multiple zeta functions, Topology Appl., 62 (1995), no. 2, 193-206. MR.1320252 (96c:57017)

[28] M. V. Subbarao and R. Sitaramachandra Rao, On some infinite series of L. J. Mordell and their analogues, Pacific J. Math., 119 (1985), no. 1, 245-255. MR.0797027 (87c:11091)

[29] T. Terasoma, Selberg integrals and multiple zeta values, Compositio Math., 133 (2002), no. 1, 1-24. MR:1918286 (2003k:11142)

[30] _ Mixed Tate motives and multiple zeta values, Invent. Math., 149 (2002), no. 2, 339-369. MR 1918675 (2003h:11073)

[31] _ Period integrals and multiple zeta values, Sūgaku, 57 (2005), no. 3, 255-266. MR2163671 (2006h:11110)

[32] L. Tornheim, Harmonic double series, Amer. J. Math., 72 (1950), 303-314. MR0034860 (11:654a)

[33] H. Tsumura, On some combinatorial relations for Tornheim's double series, Acta Arith., 105 (2002), no. 3, 239-252. MR.1931792 (2003i:11134) 
[34] - On alternating analogues of Tornheim's double series, Proc. Amer. Math. Soc., 131 (2003), no. 12, 3633-3641. MR1998168 (2004e:11102)

[35] _ Evaluation formulas for Tornheim's type of alternating double series, Math. Comp., 73 (2004), no. 245, 251-258. MR2034120 (2005d:11137)

[36] M. Waldschmidt, Valeurs zêta multiples: Une introduction, J. Théor. Nombres Bordeaux, 12 (2000), no. 2, 581-595. MR 1823204 (2002a:11106)

[37] _ Multiple polylogarithms: An introduction, in Number Theory and Discrete Mathematics (Chandigarh, 2000), Trends Math., Birkhäuser, Basel, 2002, pp. 1-12. MR.1952273 (2004d:33003)

[38] V. V. Zudilin, Algebraic relations for multiple zeta values (Russian), Uspekhi Mat. Nauk, 58 (2003), no. 1, 3-32; translation in Russian Math. Surveys, 58 (2003), no. 1, 1-29. MR.1992130 (2004k:11150)

Department of Mathematics, Zhejiang University, Hangzhou, 310027, People's RePUBLIC OF CHINA

E-mail address: xiazhou0821@hotmail.com

Department of Mathematics, Zhejiang University, Hangzhou, 310027, People's RePUBLIC OF CHINA

E-mail address: txcai@mail.hz.zj.cn

Department of Mathematics and Statistics, University of Maine, 5752 Neville Hall, Orono, Maine 04469-5752

E-mail address: bradley@math.umaine.edu

E-mail address: dbradley@member.ams.org 